\title{
Cardiac exercise imaging using a 3-tesla magnetic resonance-conditional pedal ergometer: Preliminary results in healthy volunteers and patients with known or suspected coronary artery disease
}

\author{
Agnes Mayr ${ }^{1}$, Gert Klug ${ }^{2}$, Sebastian J. Reinstadler ${ }^{2}$, Regina Esterhammer ${ }^{3}$, \\ Christian Kremser ${ }^{1}$, Klemens Mairer ${ }^{1}$, Bernhard Metzler ${ }^{2}$, Michael F. Schocke ${ }^{3}$ \\ ${ }^{1}$ University Clinic of Radiology, Medical University of Innsbruck, Austria \\ ${ }^{2}$ University Clinic of Internal Medicine III, Cardiology and Angiology, \\ Medical University of Innsbruck, Austria \\ ${ }^{3}$ Rehabilitation and University Hospital Ulm, Department of Radiology and Neuroradiology, \\ University Clinic of Ulm, Germany
}

\begin{abstract}
Background: Cardiac magnetic resonance imaging (CMR) remains underutilized as an exercise imaging modality, mostly because of the limited availability of MR-compatible exercise equipment. This study prospectively evaluates the clinical feasibility of a newly developed MR-conditional pedal ergometer for exercise CMR

Methods: Ten healthy volunteers (mean age $44 \pm 16$ years) and 11 patients (mean age $60 \pm 9$ years) with known or suspected coronary artery disease $(C A D)$ underwent rest and post-exercise cinematic $3 T$ CMR. Visual analysis of wall motion abnormalities (WMA) was rated by 2 experienced radiologists, and volumes and ejection fractions (EF) were determined. Image quality was assessed by a 4-point Likert scale for visibility of endocardial borders.

Results: Median subjective image quality of real-time cine at rest was 1 (interquartile range [IQR] $1-2)$ and $2(I Q R 2-2.5)$ for post-exercise real-time cine $(p=0.001)$. Exercise induced a significant increase in heart rate (62 [62-73] to 111 [104-143] bpm, $p<0.0001)$. Stroke volume and cardiac index increased from resting to post-exercise conditions ( $85 \pm 21$ to $101 \pm 19 \mathrm{~mL}$ and $2.9 \pm 0.7$ to $6.6 \pm$ $\pm 1.9 \mathrm{~L} / \mathrm{min} / \mathrm{m}^{2}$, respectively; both $\left.p<0.0001\right)$, driven by a reduction in end-systolic volume $(55 \pm 20$ to $42 \pm 21 \mathrm{~mL}, p<0.0001)$. Patients (2/11) with inducible regional WMA at high-resolution postexercise cine imaging revealed significant coronary artery stenosis in subsequently performed invasive coronary angiography.

Conclusions: Exercise-CMR using our newly developed $3 T$ MR-conditional pedal ergometer is clinically feasible. Imaging of both cardiac response and myocardial ischemia, triggered by dynamic stress, is rapidly conducted while the patient is near their peak heart rate. (Cardiol J 2023; 30, 2: 276-285)

Key words: feasibility study, cine magnetic resonance imaging, stroke volume, physiological stress, heart rate
\end{abstract}

\section{Introduction}

Stress cardiac magnetic resonance imaging (CMR) offers effective cardiac prognostication in patients with stable chest pain syndromes and is frequently performed using pharmacological agents $[1,2]$. Exercise stress testing, however, provides a physiological challenge to the myocardium, with

Address for correspondence: Agnes Mayr, MD, University Clinic of Radiology, Medical University of Innsbruck, Anichstrasse 35, A-6020 Innsbruck, Austria, tel: +43 512504 82622, fax: +43512 504 22758, e-mail: a.mayr@i-med.ac.at 
the ability to link physical activity to symptoms and ischemia [3-5]. CMR, while being the clinical gold standard for assessing cardiac morphology and systolic function, remains underutilized as an exercise imaging modality, mostly because of the limited availability of MR-compatible exercise equipment [6]. Several investigators demonstrated exercise stress CMR using an in-room treadmill system followed by a rapid transfer of the subject into the scanner with subsequent imaging [7-12]. On the other hand, MR-compatible equipment enabling imaging at 1.5 tesla $(\mathrm{T})$ while the patient is at peak exercise stress was shown to be feasible for the evaluation of blood flow dynamics and ventricular function in healthy volunteers [13-20]. Another proof-ofconcept study reported on a $3 \mathrm{~T}$ compatible ergometer, which allowed the assessment of cardiac morphology and function in healthy subjects [6]. Apart from safety concerns, the improvement of the signal-to-noise ratio, and subsequently the spatial and temporal resolution, makes high-field imaging particularly attractive. Due to the limited availability of MR-compatible exercise equipment for high magnetic field strengths; however, publications showing the feasibility of cardiac exercise stress testing at 3T in patients with suspected coronary artery disease (CAD) are lacking. Therefore, the purpose of this study was to evaluate a newly designed 3T conditional pedal ergometer for cardiac stress imaging using rapid CMR acquisition techniques prior to and immediately after maximal exercise stress in healthy volunteers and in patients with known or suspected CAD.

\section{Methods}

\section{Study population}

Ten healthy volunteers and 11 patients with known or suspected CAD were recruited for this single-center, prospective, feasibility study. Healthy subjects met the inclusion criteria of age between 20 and 80 years and no medical history of cardiovascular, metabolic, or neurological disease. Inclusion criteria for patients included the same age limits as for healthy volunteers and an additional referral to elective invasive coronary angiography (CAG) at the local department of cardiology based on known or suspected CAD with moderate to high pretest probability for CAD. Exclusion criteria for both healthy subjects and patients included physical exercise restrictions and general contraindications for CMR. The study complies with the ethical guidelines of the 1975 Declaration of Helsinki and was approved by the local ethics committee.
Written informed consent was obtained from all participants before inclusion into the study.

\section{Equipment and exercise protocol}

Exercise testing was conducted using a newly developed, commercially available ergometer, which is compatible with MR scanners up to 7T (Ergospect GmbH, Cardio Step Module). The pedal ergometer consists of a 2-cylinder hydraulic system connected to an air pressure system for power control. The resistance that must be overcome for movement of the exercise pedal is generated by hydraulic throttling of a piston-cylinder system. For this purpose, a hydraulic throttle valve pneumatically piloted by means of a differential pressure valve is used. A control unit regulates the air pressure, by which the hydraulic system resistance can be regulated continuously with manual force adjustment. All structural components, and power and control systems were constructed using different kinds of plastic and aluminum in order to prevent artifacts during the CMR measurements. The pedal ergometer is mounted on the scanner table, permitting supine positioning of the patient. Velcro straps were used to fix patients' feet to the pedals, and patients were in addition secured to the scanner table using a special harness in order to prevent movement artifacts. This enables CMR measurements during exercise within the isocenter of the MR scanner without any repositioning of the patient. With this setting, post-exercise imaging could be started immediately after the end of exercise. The incremental maximal exercise protocol started with pedaling against a pressure of 2 atmospheres (atm) at a cadence of 30 revolutions per minute (rpm) and progressing by 0.5 atm every 2 minutes up to volitional exhaustion, which was characterized as a significant and persistent loss of cadence ( $\geq 10 \mathrm{rpm}$ despite verbal encouragement to continue). Heart rate and work rate were recorded continuously.

\section{Cardiac magnetic resonance imaging}

Cardiac magnetic resonance imaging scans were performed on a 3T MR scanner (Magnetom Skyra, Siemens Healthineers AG). The CMR protocol included:

- Plethysmography-triggered first-pass perfusion-balanced steady-state free precession sequence (bSSFP/true fast imaging with steady-state precession [trueFISP]) at rest conditions during intravenous administration of $0.15 \mathrm{mmol} / \mathrm{kg}$ of Gd-DO3A-butriol (Gadovist $^{\oplus}$, Bayer Vital), which was admin- 
istered using an automatic injector (Spectris Injection System, Medrad) at $2 \mathrm{~mL} / \mathrm{s}$, followed by $20 \mathrm{~mL}$ of saline flush. Three short-axis slices and one long-axis slice were acquired;

- Real-time cine imaging at rest and immediately after the end of exercise, using a non-gated, free-breathing bSSFP acquisition for volumetric measurements. A short-axis stack covering the left ventricle (LV) and 3 long-axis images (a 3-chamber, a 2-chamber, and a 4-chamber slice) were acquired;

- High-resolution cine imaging post exercise using retrospectively plethysmography-triggered bSSFB bright-blood sequences with breath hold for the detection of any regional LV wall motion abnormalities (WMA). Three short-axis and 3 long-axis slices were acquired;

- Late gadolinium enhancement imaging (LGE) by using a plethysmography-triggered single-shot phase-sensitive inversion recovery (PSIR) sequence with bSSFP readout. A short-axis stack covering the LV and 3 long-axis images were acquired.

The imaging parameters of the respective sequences are shown in Table 1.

\section{Image analysis}

The overall subjective image quality of real-time cine images at rest and post-exercise condition was graded by 2 radiologists (A.M. and M.S.) with several years of experience in cardiovascular imaging (12 and 22 years, respectively), blinded to the results of each other as well as to clinical information. Subjective image quality grading was based on a 4 -point Likert scale $(1=$ excellent image quality, $2=$ good image quality, $3=$ acceptable diagnostic despite impairments by artefacts, $4=$ non-diagnostic). Volumetric evaluation of rest and post-exercise real-time cine images was performed using standard software (ARGUS, Siemens Erlangen, Germany). Contouring of LV endo- and epicardial borders was performed semi-automatically by both observers, and mean values were used for statistical analysis.

High-resolution cine images at rest and postexercise conditions were visually analyzed by both observers. Regional WMAs were defined as deterioration in regional wall motion within a myocardial segment after exercise relative to the wall motion observed during rest imaging. Thereby wall motion was labeled as hyperkinetic, normal, hypokinetic, akinetic, or dyskinetic. Myocardial ischemia was defined if $\geq 3$ out of 16 American Heart Association (AHA) segments showed new

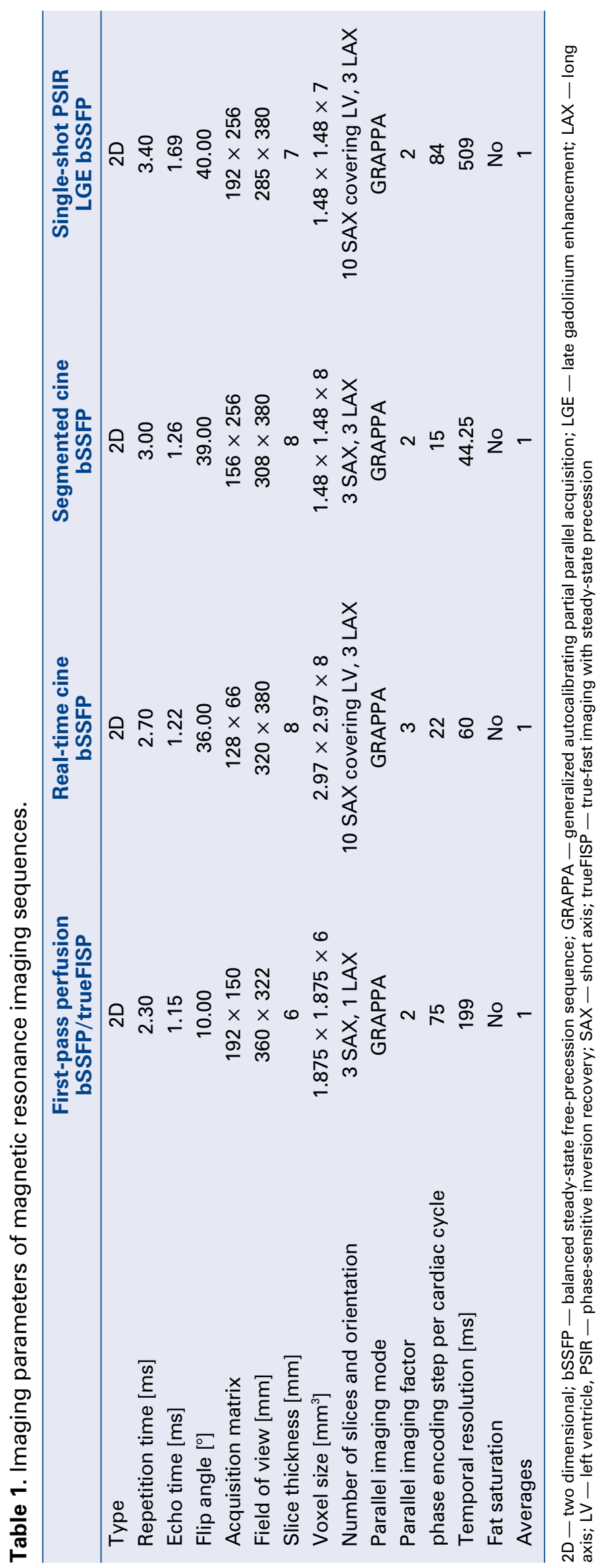


Table 2. Characteristics of the study cohort.

\begin{tabular}{lcccc}
\hline Characteristic & All subjects $(\mathbf{n}=\mathbf{2 1})$ & Healthy $(\mathbf{n}=\mathbf{1 0})$ & Patients $(\mathbf{n}=\mathbf{1 1})$ & $\mathbf{P}$ \\
\hline Age $[$ years] & $52.9 \pm 14.6$ & $44.9 \pm 16$ & $60.1 \pm 8.9$ & $\mathbf{0 . 0 1 3}$ \\
Female & $4(19 \%)$ & $1(10 \%)$ & $3(27 \%)$ & 0.512 \\
Male & $17(81 \%)$ & $9(90 \%)$ & $8(73 \%)$ & \\
Body mass index $\left[\mathrm{kg} / \mathrm{m}^{2}\right]$ & $26.5 \pm 4.1$ & $25.9 \pm 4.6$ & $27 \pm 3.7$ & 0.852 \\
Body surface area $\left[\mathrm{m}^{2}\right]$ & $1.89 \pm 0.17$ & $1.89 \pm 0.2$ & $1.9 \pm 0.13$ & 0.106 \\
Heart rate at rest $[\mathrm{bpm}][\mathrm{IQR}]$ & $62[62-73]$ & $65[60-73]$ & $67[62-77]$ & 0.973 \\
Heart rate postexercise $[\mathrm{bpm}][\mathrm{IOR}]$ & $111[104-143]$ & $125[105-166]$ & $111[100-125]$ & 0.349 \\
\hline
\end{tabular}

Data are shown as number (\%), mean \pm standard deviation; IQR — interquartile range

WMA [21]. Each observer was blinded to the ratings of the other.

\section{Statistical analysis}

Statistical analysis was conducted using Statistical Package for Social Sciences (SPSS) version 24.0 (IBM AG). Categorical variables are presented as frequencies and corresponding percentages. The Shapiro-Wilk test was applied to test for normal distribution (ND). All results are expressed as mean \pm standard deviation (SD) (if ND) or as median with interquartile range (IQR) (if not ND). Correlations of continuous variables were calculated with Pearson's or Spearman's rank test as appropriate. Differences of the recorded parameters were compared with Student's $t$ test for paired or unpaired variables (ND) or Mann-Whitney U test (if not ND). The interclass correlation coefficient (ICC) from a 2-way mixed model for single measures was used to compare image quality scores between observers. For all tests, a 2 -tailed p value $<0.05$ was considered statistically significant.

\section{Results}

Ten healthy volunteers (mean age $44.9 \pm 16$ years, 9 [90\%] males) and 11 patients (mean age $60.1 \pm 8.9$ years, 8 [73\%] male) completed the feasibility study. The mean age of the overall study cohort was $52.9 \pm 14.65$ years (17 [81\%] males). Detailed demographic characteristics of the study cohort are shown in Table 2.

Exercise testing by pedal ergometer focused on the maximum possible stress level of healthy volunteers and patients. The primary reason for stopping exercise was the insurmountable resistance level (all of the healthy volunteers, 8/11 of the patients). Two (22\%) patients reported breathlessness, and 1 patient stopped exercise because of mild angina pectoris symptoms. Twenty-four percent of the participants (5/21) met $85 \%$ of the age-predicted maximal heart rate (as calculated by $85 \% \times[220-$ age $])$, whereof $2 / 10(20 \%)$ were healthy volunteers and $3 / 11(27 \%)$ were patients. Seventy percent of the age-predicted maximal heart rate (as calculated by $70 \% \times[220-$ age $]$ ) was achieved for $71 \%(15 / 21)$ of the participants (6/10 healthy volunteers, $9 / 11$ patients).

Cardiac imaging at rest and immediately post exercise was successfully completed in all of the subjects. The mean examination time for completing the whole study protocol including localizers, first-pass-perfusion imaging, and real-time and high-resolution cine imaging at rest and post-exercise including a test run with the pedal ergometer as well as LGE imaging was $22.3 \pm 3.5$ min. Absolute exercise time using the multistage strain protocol was on average $7.8 \pm 3.2 \mathrm{~min}$. Postexercise real-time cine imaging was completed within $3 \pm 1 \mathrm{~s}$ after the end of exercise, and postexercise high-resolution cine imaging under breath hold was finished after an additional $53 \pm 8$ s.

\section{Image quality}

Median subjective image quality of real-time cine studies at rest was 1 (IQR $1-2$ ) and 2 (IQR $2-2.5)$ for post-exercise real-time cine $(\mathrm{p}=0.001)$. Images of real-time cine studies under rest and post-exercise conditions were evaluated with good to excellent inter-observer reliability $(\mathrm{ICC}=0.999$ and 0.862 , respectively, all $\mathrm{p}<0.0001$; Fig. 1). Image quality did not differ significantly between patients and healthy volunteers $(\mathrm{p}>0.05)$.

\section{Cardiac reserve and regional wall motion abnormalities post-exercise \\ Heart rate, LV ejection fraction, stroke vol- ume, as well as cardiac output and cardiac index increased significantly from rest to post-exercise}




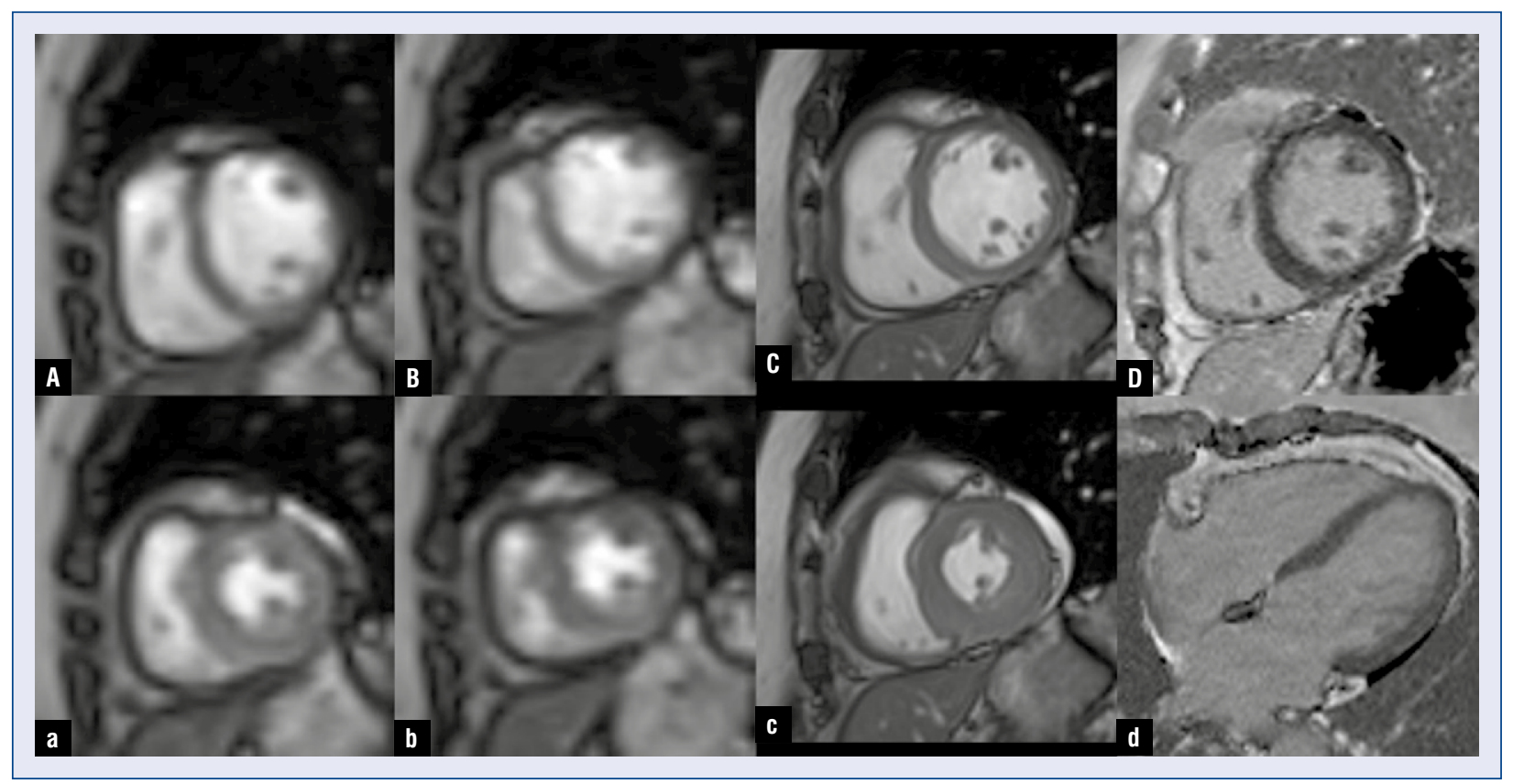

Figure 1. Representative cardiac magnetic resonanse images of a 48-year-old healthy volunteer. Left column: Real-time cine of basal short-axis slice at rest end-diastolic (A) and end-systolic (a). Second column: Real-time cine post-exercise end-diastolic (B) and end-systolic (b). Third column: high-resolution cine at rest end-diastolic (C) and end-systolic (c). Right column: late gadolinium enhancement short-axis slice (D) and 4-chamber view (d). Heart rate at rest $62 \mathrm{bpm}$, heart rate post-exercise $111 \mathrm{bpm}$.

conditions in healthy volunteers as well as patients (all $\mathrm{p}<0.0001)$. The increase in stroke volume was mediated by a reduction in end-systolic volume (ESV) in healthy volunteers and patients ( $p<0.002$ and $p<0.0001$, respectively). End-diastolic volume (EDV) did not change with exercise (all p > 0.066; Tables 3 and 4, Fig. 2).

Two of 11 patients showed inducible regional WMA on post-exercise high-resolution cine images, without evidence of any ischemic scar in the LGE sequences. One patient showed hypokinetic WMA in the anteroseptal segments at midventricular and apical LV level (4/16 AHA segments) on post-exercise cine images. Subsequent CAG of this patient revealed a $90 \%$ proximal left anterior descending artery - first diagonal artery bifurcation stenosis, resulting in percutaneous coronary intervention with stent implantation (Fig. 3). Another patient with hypokinetic WMA of the anterior segments at basal and midventricular level (3/16 AHA segments) under stress imaging conditions subsequently needed stenting of a $90 \%$ stenosis of the Ramus intermedius. All the other patients did not showed any newly regional WMA on post-exercise high-resolution cine images or any coronary stenosis requiring intervention on subsequent $\mathrm{CAG}$.

\section{Discussion}

To the best of our knowledge, this study is the first to demonstrate the feasibility of physical stress imaging by a $3 \mathrm{~T}$ compatible pedal ergometer in healthy volunteers and patients with known or suspected CAD. The main study findings are the following:

- Our newly developed 3T compatible pedal ergometer enables exercise testing in order to image cardiac responses to dynamic stress;

- The current results suggest a reliable ability to image myocardial ischemia in patients with CAD triggered by pedal ergometer exercise inside the bore of the magnet;

- Free-breathing real-time cine images acquired immediately post exercise while the patient is at their peak heart rate provides good image quality.

While CMR is rarely used for imaging cardiac exercise stress in general, the use of exercise equipment, particularly at higher magnetic field strengths, still seems to be very limited. 3T CMR, however, has become widespread and offers advantages over 1.5T for a broad range of applications, for example perfusion imaging and LGE [22, 23]. Furthermore, the optimized signal-to-noise ratio 
Table 3. Comparison of rest and post-exercise parameters of the entire study cohort.

\begin{tabular}{lccc} 
& Rest $(\mathbf{n}=\mathbf{2 1})$ & Post exercise $(\mathbf{n}=\mathbf{2 1})$ & $\mathbf{P}$ \\
\hline Heart rate $[\mathrm{bpm}][\mathrm{IQR}]$ & $62[62-73]$ & $111[104-143]$ & $\mathbf{0 . 0 0 0 1}$ \\
EF $[\%]$ & $62 \pm 9$ & $71 \pm 8$ & $\mathbf{0 . 0 0 0 1}$ \\
EDV $[\mathrm{mL}]$ & $140 \pm 32$ & $142 \pm 30$ & 0.094 \\
EDV $\left[\mathrm{mL} / \mathrm{m}^{2}\right]$ & $74 \pm 16$ & $75 \pm 16$ & 0.067 \\
ESV $[\mathrm{mL}]$ & $55 \pm 20$ & $42 \pm 21$ & $\mathbf{0 . 0 0 0 1}$ \\
ESV $\left[\mathrm{mL} / \mathrm{m}^{2}\right]$ & $29 \pm 11$ & $22 \pm 12$ & $\mathbf{0 . 0 0 0 1}$ \\
SV $[\mathrm{mL}]$ & $85 \pm 21$ & $101 \pm 19$ & $\mathbf{0 . 0 0 0 1}$ \\
SV $\left[\mathrm{mL} / \mathrm{m}^{2}\right]$ & $45 \pm 10$ & $53 \pm 9$ & $\mathbf{0 . 0 0 0 1}$ \\
CO $[\mathrm{L} / \mathrm{min}]$ & $5.6 \pm 1.5$ & $12.5 \pm 4$ & $\mathbf{0 . 0 0 0 1}$ \\
$\mathrm{Cl}\left[\mathrm{L} / \mathrm{min} / \mathrm{m}^{2}\right]$ & $2.9 \pm 0.7$ & $6.6 \pm 1.9$ & $\mathbf{0 . 0 0 0 1}$ \\
\hline
\end{tabular}

Data are shown as mean \pm standard deviation; $\mathrm{Cl}$ — cardiac index; $\mathrm{CO}$ - cardiac output; EDV — end-diastolic volume; EF — ejection fraction; ESV - end-systolic volume; IQR — interquartile range; SV — stroke volume

Table 4. Comparison of rest and post-exercise parameters for healthy volunteers and patients with known or suspected coronary artery disease (CAD).

\begin{tabular}{|c|c|c|c|}
\hline & Rest conditions & Post-exercise conditions & $\mathbf{P}$ \\
\hline \multicolumn{4}{|c|}{ Healthy volunteers $(n=10)$} \\
\hline Heart rate [bpm] [IOR] & $65[60-73]$ & 125 [105-167] & 0.0001 \\
\hline $\mathrm{EF}[\%]$ & $64 \pm 8$ & $73 \pm 6$ & 0.005 \\
\hline EDV $[\mathrm{mL}]$ & $136 \pm 34$ & $138 \pm 40$ & 0.242 \\
\hline $\mathrm{EDV}\left[\mathrm{mL} / \mathrm{m}^{2}\right]$ & $72 \pm 15$ & $73 \pm 15$ & 0.186 \\
\hline $\mathrm{ESV}[\mathrm{mL}]$ & $48 \pm 14$ & $36 \pm 13$ & 0.002 \\
\hline $\mathrm{ESV}\left[\mathrm{mL} / \mathrm{m}^{2}\right]$ & $25 \pm 7$ & $19 \pm 7$ & 0.002 \\
\hline $\mathrm{SV}[\mathrm{mL}]$ & $88 \pm 28$ & $102 \pm 24$ & 0.0001 \\
\hline $\mathrm{SV}\left[\mathrm{mL} / \mathrm{m}^{2}\right]$ & $46 \pm 13$ & $54 \pm 11$ & 0.0001 \\
\hline $\mathrm{CO}[\mathrm{L} / \mathrm{min}]$ & $5.6 \pm 1.9$ & $13 \pm 5$ & 0.0001 \\
\hline $\mathrm{Cl}\left[\mathrm{L} / \mathrm{min} / \mathrm{m}^{2}\right]$ & $2.9 \pm 0.9$ & $7 \pm 2.5$ & 0.0001 \\
\hline \multicolumn{4}{|c|}{ Patients with known or suspected CAD } \\
\hline Heart rate [bpm] [IQR] & 62 [62-77] & $111[100-125]$ & 0.0001 \\
\hline EF [\%] & $59 \pm 9$ & $70 \pm 10$ & 0.0001 \\
\hline $\mathrm{EDV}[\mathrm{mL}]$ & $145 \pm 30$ & $146 \pm 29$ & 0.263 \\
\hline $\mathrm{EDV}\left[\mathrm{mL} / \mathrm{m}^{2}\right]$ & $77 \pm 16$ & $76 \pm 17$ & 0.244 \\
\hline $\mathrm{ESV}[\mathrm{mL}]$ & $61 \pm 24$ & $47 \pm 26$ & 0.0001 \\
\hline $\mathrm{ESV}\left[\mathrm{mL} / \mathrm{m}^{2}\right]$ & $32 \pm 13$ & $25 \pm 15$ & 0.0001 \\
\hline $\mathrm{SV}[\mathrm{mL}]$ & $84 \pm 14$ & $100 \pm 14$ & 0.0001 \\
\hline $\mathrm{SV}\left[\mathrm{mL} / \mathrm{m}^{2}\right]$ & $44 \pm 7$ & $53 \pm 7$ & 0.0001 \\
\hline $\mathrm{CO}$ [L/min] & $5.6 \pm 1$ & $11.7 \pm 1.8$ & 0.0001 \\
\hline $\mathrm{Cl}\left[\mathrm{L} / \mathrm{min} / \mathrm{m}^{2}\right]$ & $2.9 \pm 0.6$ & $6.2 \pm 0.9$ & 0.0001 \\
\hline
\end{tabular}

Data are shown as mean \pm standard deviation. $\mathrm{Cl}$ - cardiac index; $\mathrm{CO}$ - cardiac output; EDV — end-diastolic volume; $\mathrm{EF}$ - ejection fraction; $\mathrm{ESV}$ — end-systolic volume; IQR — interquartile range; SV — stroke volume

and image resolution at $3 \mathrm{~T}$ compared to $1.5 \mathrm{~T}$ permit the detection of small and slight volumetric/ /structural alterations [24]. On the other hand, for CMR at higher magnetic field strength, greater ef- forts are needed to prevent artefacts particularly in the setting of ECG triggering $[25,26]$. In addition, stress imaging may worsen heart rate variability and arrhythmias, leading to skipped cardiac cycles 


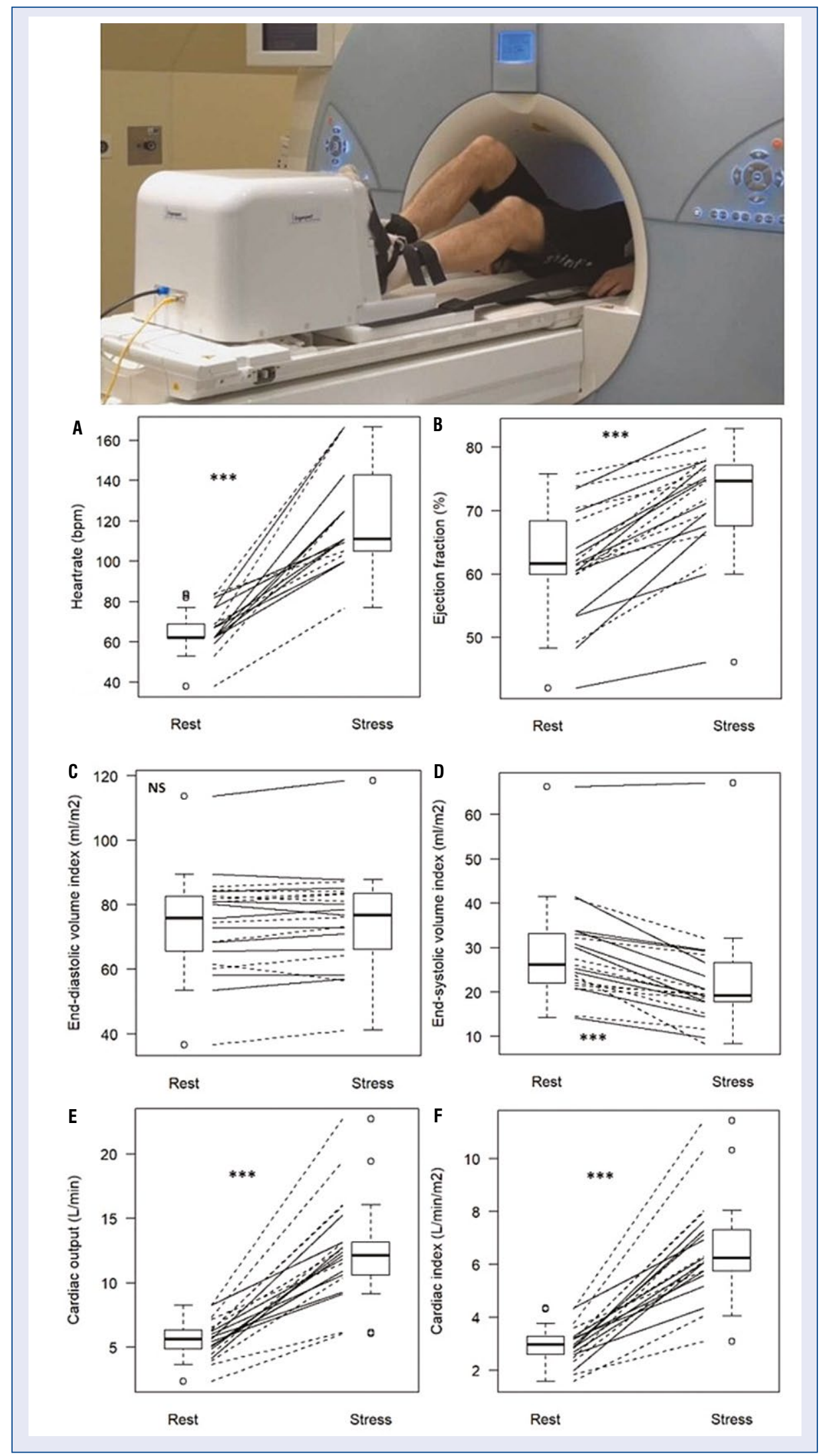

Figure 2. Graphs show variation of heart rate at rest and immediately post-exercise (A), ejection fraction (B), end-diastolic volume index (C), end-systolic volume index (D), cardiac output (E), cardiac index (F), and as assessed by free-breathing real-time cine imaging. Error bars $=$ mean \pm standard deviation; ${ }^{* *} p<0.0001$; NS — non-significant. 


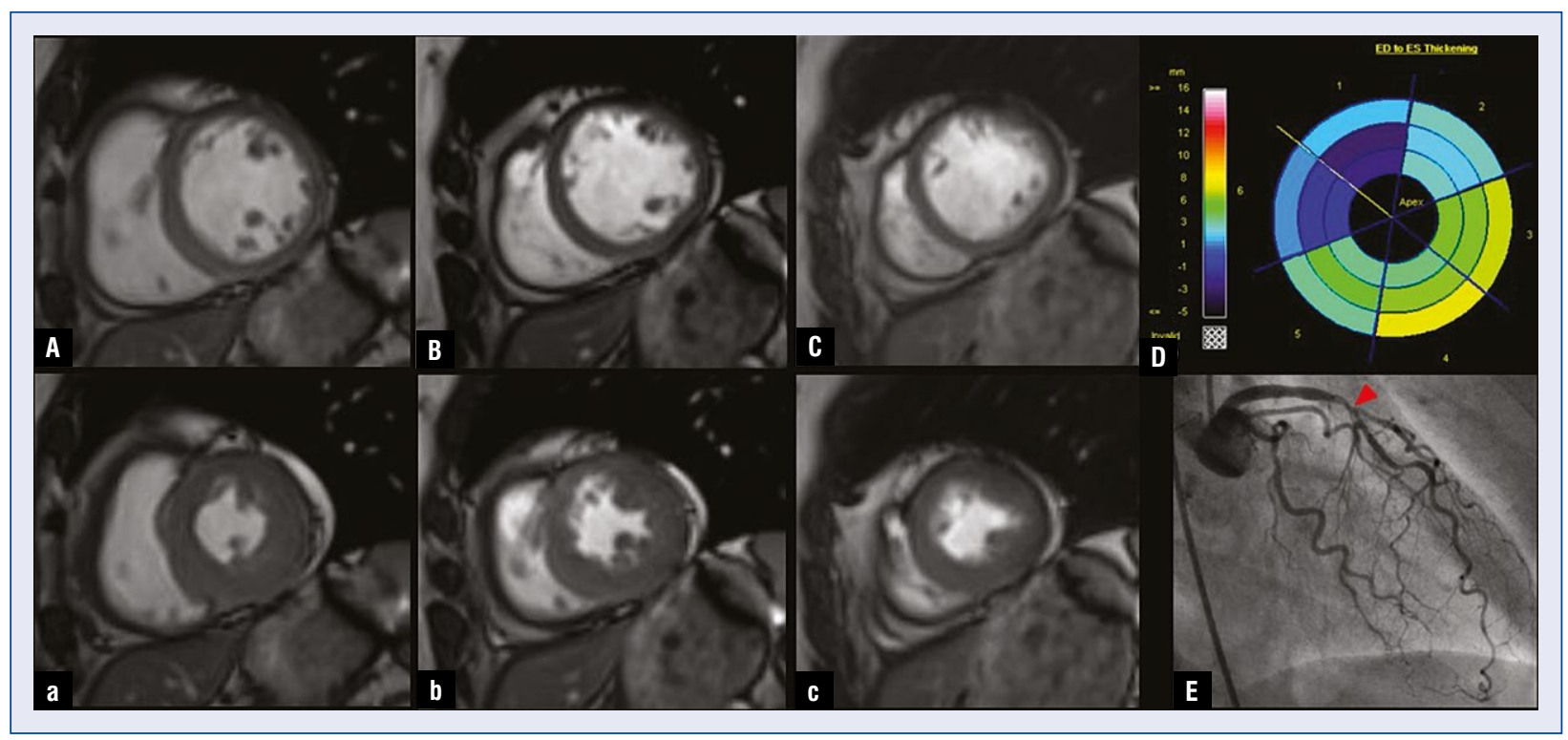

Figure 3. Representative cardiac magnetic resonance imaging images of a 65-year-old female patient with suspected coronary artery disease. Upper row: High-resolution cine post-exercise end-diastolic on a basal short axis slice (A), midventricular short-axis slice (B), and apical short-axis slice (C). Lower row (a-c): High-resolution cine post-exercise end-systolic frames show hypokinetic wall motion abnormality in the anteroseptal segments of midventricular (b) and apical slice of the left ventricle (c), as illustrated by reduced end-diastolic to end-systolic wall thickening in bullseye diagram (D). Percutaneous coronary angiography on the following day depicted a $90 \%$ proximal left anterior descending artery - first diagonal artery bifurcation stenosis (E, arrowhead), which was intervened by stent implantation. Heart rate at rest imaging $59 \mathrm{bpm}$, heart rate at post-exercise imaging $125 \mathrm{bpm}$.

with the potential of losing diagnostically important information. Consequently, several study groups are working on alternative gating methods like Doppler ultrasound, acoustic triggering, or selfgating [27-31]. Another widespread technique, the feasibility of which was shown earlier [32-34], is peripheral pulse gating (photoplethysmography), as was used in the current study.

It was shown previously that exercising outside of the MR scanner with subsequent fast transition into the scanner for image acquisition can be performed within $60 \mathrm{~s}$ of peak exercise [6-12]. However, hemodynamic recovery occurs rapidly, and caution is required when interpreting data acquired more than $4 \mathrm{~s}$ post exercise. This is especially important in the younger, healthy population, but also clinical patients show a comparable decline in heart rate $[6,9]$. Our results demonstrate that post-exercise real-time cine imaging can be completed within $3 \pm 1 \mathrm{~s}$ after the end of exercise to comprehensively image cardiac responses to dynamic stress. Regarding imaging of myocardial ischemia, exercise-induced regional WMA is known to begin to diminish immediately following cessation of exercise [35]. For this reason, we used rapid post-exercise, high-resolution cine imaging in this study, in order to perform this imaging step within the first minute after exercise cessation. Thus, newly emerged regional WMA could be detected in 2 patients who subsequently underwent coronary intervention. It is necessary to confirm these results in a future study with a significantly larger number of patients.

In line with previous reports, our data suggest that supine positioning within the bore of the magnet in combination with leg elevation can produce near maximal EDVs because both healthy subjects and patients showed no EDV reserve during supine exercise. Along with an increase in heart rate, cardiac reserve was mediated by a significant reduction in ESV from rest to post-exercise conditions. This resulted in an increase of the cardiac index for healthy people and patients by a factor of more than 2. In fact, conventional upright cycling showed major ESV changes compared to supine exercise, reflecting recruitment of a greater muscle mass [12]. However, prior investigations using an MR-compatible ergometer showed reduced metabolic demand by supine exercising $[6,16]$. Moreover, the stepwise resistance approach used in our study enables a steady-state workload. Because the heart rate has the strongest association 
with myocardial work of all non-invasive measures, its quantification is crucial during cardiac function studies [35]. Admittedly, only $24 \%$ of the participants in the current study met $85 \%$ of age-predicted maximal heart rate whereas $71 \%$ achieved $70 \%$ of age-predicted maximal heart rate. However, our exercise testing by pedal ergometer focused on the maximum possible stress level of participants, with an insurmountable resistance level being the primary reason for aborting the exercise (18/21 participants). Maximum heart rates achieved in this way are at a comparable level to previous MR studies using supine exercise testing while still allowing reliable synchronization of MR data acquisition by peripheral pulse gating $[6,13$, 36-38] as well as good subjective image quality for post-exercise real-time Cine images.

\section{Conclusions}

Our findings suggest that exercise stress CMR using the proposed 3T MR conditional pedal ergometer is clinically feasible. It enables reliable imaging of functional response in healthy subjects as well as myocardial ischemia triggered by dynamic stress in patients with known or suspected CAD. For the latter, in particular, confirmation of this finding in larger trials is essential.

\section{Funding}

This study was supported by K-Regio, project number WIF-275-01-00002/01-0042.

\section{Conflict of interest: None declared}

\section{References}

1. Kwong RY, Ge Y, Steel K, et al. Cardiac magnetic resonance stress perfusion imaging for evaluation of patients with chest pain. J Am Coll Cardiol. 2019; 74(14): 1741-1755, doi: 10.1016/j.jacc.2019.07.074, indexed in Pubmed: 31582133.

2. Nagel E, Berry C, Nagel E, et al. MR-INFORM Investigators. Magnetic Resonance Perfusion or Fractional Flow Reserve in Coronary Disease. N Engl J Med. 2019; 380(25): 2418-2428, doi: 10.1056/NEJMoa1716734, indexed in Pubmed: 31216398.

3. Bourque JM, Beller GA. Value of exercise ECG for risk stratification in suspected or known CAD in the era of advanced imaging technologies. JACC Cardiovasc Imaging. 2015; 8(11): 1309-1321, doi: 10.1016/j.jcmg.2015.09.006, indexed in Pubmed: 26563861.

4. Demir OM, Bashir A, Marshall K, et al. Comparison of clinical efficacy and cost of a cardiac imaging strategy versus a traditional exercise test strategy for the investigation of patients with suspected stable coronary artery disease. Am J Cardiol. 2015; 115(12): 1631-1635, doi: 10.1016/j.amjcard.2015.03.005, indexed in Pubmed: 25910528.

5. Ferket BS, Genders TSS, Colkesen EB, et al. Systematic review of guidelines on imaging of asymptomatic coronary artery dis- ease. J Am Coll Cardiol. 2011; 57(15): 1591-1600, doi: 10.1016/j. jacc.2010.10.055, indexed in Pubmed: 21474039.

6. Beaudry RI, Samuel TJ, Wang J, et al. Exercise cardiac magnetic resonance imaging: a feasibility study and meta-analysis. Am J Physiol Regul Integr Comp Physiol. 2018; 315(4): R638-R645, doi: 10.1152/ajpregu.00158.2018, indexed in Pubmed: 29949409.

7. Foster EL, Arnold JW, Jekic M, et al. MR-compatible treadmill for exercise stress cardiac magnetic resonance imaging. Magn Reson Med. 2012; 67(3): 880-889, doi: 10.1002/mrm.23059, indexed in Pubmed: 22190228.

8. Jekic M, Foster EL, Ballinger MR, et al. Cardiac function and myocardial perfusion immediately following maximal treadmill exercise inside the MRI room. J Cardiovasc Magn Reson. 2008; 10: 3, doi: 10.1186/1532-429X-10-3, indexed in Pubmed: 18272005.

9. Raman SV, Dickerson JA, Mazur W, et al. Diagnostic performance of treadmill exercise cardiac magnetic resonance: the prospective, multicenter exercise CMR's accuracy for cardiovascular stress testing (EXACT) trial. J Am Heart Assoc. 2016; 5(8), doi: 10.1161/JAHA.116.003811, indexed in Pubmed: 27543308.

10. Sukpraphrute B, Drafts BC, Rerkpattanapipat P, et al. Prognostic utility of cardiovascular magnetic resonance upright maximal treadmill exercise testing. J Cardiovasc Magn Reson. 2015; 17: 103, doi: 10.1186/s12968-015-0208-z, indexed in Pubmed: 26608545.

11. Thavendiranathan P, Dickerson JA, Scandling D, et al. Comparison of treadmill exercise stress cardiac MRI to stress echocardiography in healthy volunteers for adequacy of left ventricular endocardial wall visualization: a pilot study. J Magn Reson Imaging. 2014; 39(5): 1146-1152, doi: 10.1002/jmri.24263, indexed in Pubmed: 24123562.

12. Lafountain RA, da Silveira JS, Varghese J, et al. Cardiopulmonary exercise testing in the MRI environment. Physiol Meas. 2016; 37(4): N11-N25, doi: 10.1088/0967-3334/37/4/N11, indexed in Pubmed: 26987361.

13. Gusso S, Salvador C, Hofman P, et al. Design and testing of an MRI-compatible cycle ergometer for non-invasive cardiac assessments during exercise. Biomed Eng Online. 2012; 11: 13, doi: 10.1186/1475-925X-11-13, indexed in Pubmed: 22423637.

14. Heiberg J, Asschenfeldt B, Maagaard M, et al. Dynamic bicycle exercise to assess cardiac output at multiple exercise levels during magnetic resonance imaging. Clin Imaging. 2017; 46: 102-107, doi: 10.1016/j.clinimag.2017.07.010, indexed in Pubmed: 28778011.

15. La Gerche A, Claessen G, Van de Bruaene A, et al. Cardiac MRI: a new gold standard for ventricular volume quantification during high-intensity exercise. Circ Cardiovasc Imaging. 2013; 6(2): 329-338, doi: 10.1161/CIRCIMAGING.112.980037, indexed in Pubmed: 23258478.

16. Le TT, Bryant JA, Ting AE, et al. Assessing exercise cardiac reserve using real-time cardiovascular magnetic resonance. J Cardiovasc Magn Reson. 2017; 19(1): 7, doi: 10.1186/s12968017-0322-1, indexed in Pubmed: 28110638.

17. Oosterhof T, Tulevski II, Roest AAW, et al. Disparity between dobutamine stress and physical exercise magnetic resonance imaging in patients with an intra-atrial correction for transposition of the great arteries. J Cardiovasc Magn Reson. 2005; 7(2): 383-389, doi: 10.1081/jcmr-200053454, indexed in Pubmed: 15881518.

18. Pflugi S, Roujol S, Akçakaya M, et al. Accelerated cardiac MR stress perfusion with radial sampling after physical exercise with an MR-compatible supine bicycle ergometer. Magn Reson Med. 2015; 74(2): 384-395, doi: 10.1002/mrm.25405, indexed in Pubmed: 25105469. 
19. Steding-Ehrenborg K, Jablonowski R, Arvidsson PM, et al. Moderate intensity supine exercise causes decreased cardiac volumes and increased outer volume variations: a cardiovascular magnetic resonance study. J Cardiovasc Magn Reson. 2013; 15: 96, doi: 10.1186/1532-429X-15-96, indexed in Pubmed: 24156367.

20. Weber TF, von Tengg-Kobligk H, Kopp-Schneider A, et al. High-resolution phase-contrast MRI of aortic and pulmonary blood flow during rest and physical exercise using a MRI compatible bicycle ergometer. Eur J Radiol. 2011; 80(1): 103-108, doi: 10.1016/j.ejrad.2010.06.045, indexed in Pubmed: 20674204.

21. Knuuti J, Wijns W, Saraste A, et al. ESC Scientific Document Group. 2019 ESC Guidelines for the diagnosis and management of chronic coronary syndromes. Eur Heart J. 2020; 41(3): 407-477, doi: 10.1093/eurheartj/ehz425, indexed in Pubmed: 31504439.

22. Cheng ASH, Pegg TJ, Karamitsos TD, et al. Cardiovascular magnetic resonance perfusion imaging at 3-tesla for the detection of coronary artery disease: a comparison with 1.5-tesla. J Am Coll Cardiol. 2007; 49(25): 2440-2449, doi: 10.1016/j. jacc.2007.03.028, indexed in Pubmed: 17599608.

23. McGee KP, Debbins JP, Boskamp EdB, et al. Cardiac magnetic resonance parallel imaging at 3.0 Tesla: technical feasibility and advantages. J Magn Reson Imaging. 2004; 19(3): 291-297, doi: 10.1002/jmri.20015, indexed in Pubmed: 14994296.

24. Rajiah P, Bolen MA. Cardiovascular MR imaging at 3 T: opportunities, challenges, and solutions. Radiographics. 2014; 34(6): 1612-1635, doi: 10.1148/rg.346140048, indexed in Pubmed: 25310420

25. Sievers B, Wiesner M, Kiria N, et al. Influence of the trigger technique on ventricular function measurements using 3-Tesla magnetic resonance imaging: comparison of ECG versus pulse wave triggering. Acta Radiol. 2011; 52(4): 385-392, doi: 10.1258/ ar.2011.100505, indexed in Pubmed: 21498278.

26. Spicher N, Kukuk M, Maderwald S, et al. Initial evaluation of prospective cardiac triggering using photoplethysmography signals recorded with a video camera compared to pulse oximetry and electrocardiography at 7T MRI. Biomed Eng Online. 2016; 15(1): 126, doi: 10.1186/s12938-016-0245-3, indexed in Pubmed: 27881126 .

27. Becker M, Frauenrath T, Hezel F, et al. Comparison of left ventricular function assessment using phonocardiogram- and electrocardiogram-triggered 2D SSFP CINE MR imaging at $1.5 \mathrm{~T}$ and 3.0 T. Eur Radiol. 2010; 20(6): 1344-1355, doi: 10.1007/ s00330-009-1676-z, indexed in Pubmed: 20013275.
28. Frauenrath T, Hezel F, Renz W, et al. Acoustic cardiac triggering: a practical solution for synchronization and gating of cardiovascular magnetic resonance at 7 Tesla. J Cardiovasc Magn Reson. 2010; 12: 67, doi: 10.1186/1532-429X-12-67, indexed in Pubmed: 21080933.

29. Kording F, Schoennagel B, Lund G, et al. Doppler ultrasound compared with electrocardiogram and pulse oximetry cardiac triggering: A pilot study. Magn Reson Med. 2015; 74(5): 1257-1265, doi: 10.1002/mrm.25502, indexed in Pubmed: 25359183.

30. Kording F, Yamamura J, Lund G, et al. Doppler ultrasound triggering for cardiovascular MRI at 3T in a healthy volunteer study. Magn Reson Med Sci. 2017; 16(2): 98-108, doi: 10.2463/mrms. mp.2015-0104, indexed in Pubmed: 27001390.

31. Zhang X, Xie G, Lu Na, et al. 3D self-gated cardiac cine imaging at 3 Tesla using stack-of-stars bSSFP with tiny golden angles and compressed sensing. Magn Reson Med. 2019; 81(5): 3234-3244, doi: 10.1002/mrm.27612, indexed in Pubmed: 30474151.

32. Allen J. Photoplethysmography and its application in clinical physiological measurement. Physiol Meas. 2007; 28(3): R1-39, doi: 10.1088/0967-3334/28/3/R01, indexed in Pubmed: 17322588.

33. Humphreys K, Ward T, Markham C. Noncontact simultaneous dual wavelength photoplethysmography: a further step toward noncontact pulse oximetry. Rev Sci Instrum. 2007; 78(4): 044304, doi: 10.1063/1.2724789, indexed in Pubmed: 17477684 .

34. Kamshilin AA, Nippolainen E, Sidorov IS, et al. A new look at the essence of the imaging photoplethysmography. Sci Rep. 2015; 5: 10494, doi: 10.1038/srep10494, indexed in Pubmed: 25994481.

35. Nelson RR, Gobel FL, Jorgensen CR, et al. Hemodynamic predictors of myocardial oxygen consumption during static and dynamic exercise. Circulation. 1974; 50(6): 1179-1189, doi: 10.1161/01. cir.50.6.1179, indexed in Pubmed: 4430113.

36. Gusso S, Pinto T, Baldi JC, et al. Exercise training improves but does not normalize left ventricular systolic and diastolic function in adolescents with type 1 diabetes. Diabetes Care. 2017; 40(9): 1264-1272, doi: 10.2337/dc16-2347, indexed in Pubmed: 28720592.

37. Gusso S, Pinto TE, Baldi JC, et al. Diastolic function is reduced in adolescents with type 1 diabetes in response to exercise. Diabetes Care. 2012; 35(10): 2089-2094, doi: 10.2337/dc11-2331, indexed in Pubmed: 22773700.

38. Pinto TE, Gusso S, Hofman PL, et al. Systolic and diastolic abnormalities reduce the cardiac response to exercise in adolescents with type 2 diabetes. Diabetes Care. 2014; 37(5): 1439-1446, doi: 10.2337/dc13-2031, indexed in Pubmed: 24574351. 